\title{
Evaluación de la convivencia y percepción de conflictos en estudiantes de enseñanza secundaria en un centro educativo concertado de Málaga (España). La mediación escolar como respuesta
}

\author{
Evaluation of coexistence and perception of conflicts in secondary school students in a \\ state-subsidised school in Malaga (Spain). School mediation as an answer
}

\begin{abstract}
GONZALEZ-SODIS, José L. ${ }^{1}$
LEIVA, Juan J. ${ }^{2}$

MATAS, Antonio ${ }^{3}$

Resumen:

La conflictividad es un problema educativo y social de primer orden. En esta investigación se ha analizado la percepción de conflictos en estudiantes de una escuela del sur de España. La muestra no probabilística fue de 220 escolares. Se aplicó el CUVE3-ESO. Del análisis descriptivo-comparativo de grupos en función de género y curso se desprende la existencia de diferencias perceptivas de la conflictividad. Se concluye con la necesidad de promover la mediación escolar para mejorar el clima de convivencia.
\end{abstract}

Palabras claves: convivencia. conflictos. percepción. mediación

\begin{abstract}
Conflict is a major educational and social problem. This research has analyzed the perception of conflict in students at a school in southern Spain. The non-probabilistic sample was 220 students. The CUVE3ESO was applied. From the descriptive-comparative analysis of groups according to gender and grade, the existence of differences in the perception of conflict can be deduced. The conclusion is that it is necessary to promote school mediation to improve the climate of coexistence.

Keywords: coexistence. conflicts. perception. mediation
\end{abstract}

\section{Introducción}

La convivencia escolar es un elemento pedagógico de primer orden en la configuración emergente de propuestas que pretenden ser holísticas e inclusivas que en la década de los 90 emergió como una perspectiva prometedora para abordar una problemática relativa a la vida compartida en las escuelas. En los últimos años se ha venido implementando un buen número de programas y proyectos de mejora de la convivencia y de prevención de la

\footnotetext{
${ }^{1}$ Licenciado en Ciencias de la Educación. Licenciado en Historia. Máster en mediación de conflictos. Universidad de Málaga (España). sodis@uma.es 2 Profesor Titular del Departamento de Didáctica y Organización Escolar de la Facultad de Ciencias de la Educación de la Universidad de Málaga (España). jjleiva@uma.es

${ }^{3}$ Profesor Titular del Departamento de Teoría e Historia de la Educación y Métodos de Investigación y Diagnóstico en Educación de la Facultad de Ciencias de la Educación de la Universidad de Málaga (España). amatas@uma.es
} 
violencia escolar, promoviendo un cambio significativo desde el punto de vista de la cultura institucional y organizacional de los centros escolares (Andrades-Moya, 2020; Costa et al., 2020). Argumenta Arón et al. (2017) que la buena convivencia es un elemento inseparable de la calidad educativa y que emerge del buen clima ambiental que los centros educativos públicos, concertados y privados experimentan. El término convivencia ha generado cierta confusión como han afirmado Fierro-Evans y Carbajal-Padilla, (2019) y lo clarifica Uruñuela, (2018) diferenciando la convivencia en positivo y la convivencia positiva. Si solo procuramos una convivencia positiva supondrá planteamientos pedagógicos que reaccionan a lo que está sucediendo y que van por detrás de acontecimientos que no proyectan nada en el plano educativo. Por el contrario, la convivencia escolar en positivo debe postular una concordia sana, democrática, pacífica, no violenta, inclusiva, respetuosa con los derechos humanos, términos que reflejan las diversas miradas desde las cuales se debe abordar (Ascorra et al., 2016). El entorno escolar debe involucrarse en la cosecha de la convivencia en positivo, entendida como posicionamiento proactivo que implica la adquisición de actitudes, habilidades, y valores imprescindibles en una sociedad democrática construida sobre la paz y la no violencia. Convivir en positivo es tomar conciencia de que vivimos con otras personas y ello supone, como decimos, la puesta en práctica de competencias socioemocionales de mejora de la gestión de los conflictos en el contexto escolar, proponiendo la ciudadanía activa como elemento esencial educativo que permite entender la situaciones de conflictividad social y su transformación a través de la solución dialogada (Sánchez et al., 2019). La sociedad demanda que la experiencia pedagógica desarrollada por los docentes en las escuelas proporcione personas capacitadas y formadas en la paz y la no violencia. Ya lo planteaba Delors en su informe (1996) como uno de los retos y pilares educativos de la educación del siglo XXI. La educación persigue el desarrollo integral de los chicos y chicas, no solo en su faceta cognitiva, sino además un desarrollo en su vertiente social, aspecto este, que repercute en el bienestar de la comunidad educativa y por ende en la sociedad. Se le exige a la escuela también, que genere condiciones democráticas luchando contra la anomia social y la falta de compromiso ciudadano (Vidal et al., 2019). Rubricamos, que un buen clima educativo encierra indicadores tales como, compartir espacios colaborativos y de conversación, respeto, aceptación de las diferencias, participación e inclusividad. Por ello, aprender a convivir se está convirtiendo en uno de los parámetros más relevantes para las comunidades educativas, articulándose todo un complejo conjunto de medidas de prevención y de intervención para el fomento de la cultura de paz, como mecanismo para la convivencia humana intercultural y globalizada (Novoa et al., 2020).

Ahora bien, esta convivencia escolar puede ser alterada desde múltiples aristas y con variadas tipologías conflictivas. Diversas investigaciones muestran los distintos tipos de violencia escolar en los entornos educativos y que a veces son calificadas como violencia de baja intensidad (Brandoni, 2017, Vizcarra et al., 2018, Medina \& Villarreal, 2019, Garaigordobil, 2019, Zepeda, 2020).

Las manifestaciones de la conflictividad en el contexto educativo no son un problema episódico, más bien son catalogadas en ocasiones como un problema estructural y por ello debe ser abordado con medidas pedagógicas, efectivas y concluyentes. Generalmente, los orígenes de la violencia escolar atiende a fuentes multicausales (Uruñuela, 2018) y no solo son de orden físico, sino también de orden psicológico por lo que suelen repercutir de manera muy negativa en los jóvenes que manifiestan una endeble personalidad y baja autoestima (Ormart, 2019). Así pues, decimos que existe un conflicto escolar cuando una conducta u omisión de esta, de manera intencionada causa un daño o un perjuicio a otro miembro de la comunidad educativa (Álvarez-García et al., 2008). Atendiendo a la clasificación de Dobarro et al. (2016), las conductas indeseables y violentas que alteran la convivencia y a las que los docentes se enfrentan a diario, se podrían encuadrar en las siguientes categorías: la violencia física, la violencia verbal, la exclusión social, la disrupción en el aula y la violencia a través de las tecnologías de la información y de la comunicación (TIC). 
La violencia es un comportamiento culturalmente aprendido que tiene direccionalidad y persigue un fin: controlar, imponer, manipular o intimidar (Campos et al., 2015). El abuso de poder y la desigualdad son las bases de esta violencia escolar (Torrego, 2006, Garaigordobil \& Oñederra, 2010).

Se podría afirmar, que la violencia física directa es aquella en la que existe algún tipo de contacto físico, que incluye peleas, puñetazos, empujones, patadas, etc. Mientras que la violencia física indirecta, causa el daño actuando sobre las pertenencias o el material de trabajo de la víctima o del centro, por ejemplo, hurtos, destrozos o esconder objetos de otros miembros de la comunidad educativa (Campos et al., 2015, Estrada \& Mamani, 2020). Estudios como el realizado por Zúñiga et al., (2019), corroboran la existencia de este tipo de violencia desde los cursos de enseñanza primaria, especialmente en los últimos cursos con niños y niñas de edades comprendidas entre los 10 y 12 años.

En lo referente a la violencia verbal, se ha de señalar que es aquella en la que el daño se causa mediante el mensaje oral o escrito, es decir, insultos, motes, rumores o hablar mal de alguien. También en este caso, tal distinción se refiere al hecho de que la acción se realice a la cara, violencia verbal directa por medio del insulto o a las espaldas del compañero o compañera, violencia verbal indirecta (Nieto et al., 2018). Es muy significativa que este tipo de violencia está siendo cada vez más alarmante en las relaciones de género, así lo detallan algunos estudios al respecto (Domínguez-Alonso et al., 2019).

Los indicadores de la exclusión social son muy variados y conocidos, renta familiar, estudios de los padres, barrio, religión, nacionalidad, aspecto físico, etc. (Pachter et al., 2010 \& Brandoni, 2017). Un estudio realizado por RizoArias (2019), manifiesta analizando los datos obtenidos, que estos indicadores son elementos continuadores de la exclusión y de la segregación educativa. Dichas situaciones de exclusión pueden darse tanto dentro del aula, durante la ejecución de las tareas académicas, como fuera de ella, ignorando o excluyendo a compañeros o compañeras de los juegos o del grupo de pares durante los recreos e incluso en redes sociales.

Las conductas disruptivas son motivo de preocupación en el mundo educativo (Jurado \& Justiniano, 2016 \& Jurado de los Santos et al., 2020). Las causas implícitas en las conductas disruptivas son también en general muy variadas, y en particular en los trastornos de conducta de algunos discentes. La disrupción en el aula induce a pensar en comportamientos con los que el alumnado dificulta al docente impartir su docencia y al resto de compañeros interesados seguirla con aprovechamiento. Hablar o levantarse del asiento durante una explicación sin pedir permiso, hablar interrumpiendo, etc. (Hulac \& Benson, 2010), son ejemplos conocidos. Consecuencias negativas acarrean estas conductas para el desempeño del proceso de enseñanza-aprendizaje, repercutiendo en el bajo rendimiento académico e incluso en el fracaso escolar. La disrupción en el aula es quizá el conflicto más doméstico en las aulas de los centros educativos, es un comportamiento considerado violento y casi siempre intencionado.

En penúltima instancia, la violencia a través del uso inadecuado de las TIC, incluye comportamientos violentos a través de medios electrónicos, principalmente con la telefonía móvil y generalmente a través de las redes sociales en Internet (Tokunaga, 2010). Según Dobarro et al. (2016), dichos comportamientos violentos pueden adoptar formas variadas, por ejemplo, envío de mensajes dañinos en redes sociales a través del teléfono móvil, tabletas u ordenadores con programas de mensajería instantánea. Grabar a un compañero o compañera mientras está siendo agredido con intención de publicar la agresión o colgar fotografías de mal gusto en Instagram u otra red social frecuentada por los jóvenes. Se ha dado el caso de grabar a un profesor o profesora con la misma intención de subirlas a la red para que en poco tiempo se hagan virales. Según estudios realizados últimamente la violencia TIC está arraigando en la juventud y con mayor impacto en las chicas (Dominguez Alonso \& Portela Pino, 2020).

Generalmente, el estudio de la violencia escolar se ha centrado, casi siempre, en la violencia que surge entre estudiantes. Sin embargo, resulta también significativo considerar la relación entre los dos principales agentes 
implicados en el educativo: el docente y el alumnado. En este sentido, siempre se han analizado los comportamientos violentos que el alumnado puede desarrollar dirigidos hacia el profesorado. Del mismo modo, el alumnado puede percibir como violentas ciertas conductas del profesorado, por ejemplo, mostrar preferencias, tener manía, ignorar, insultar o burlarse de los alumnos. Teniendo en cuenta el marco teórico esbozado, se ha planteado en este estudio, como objetivo general determinar cuál es la situación de convivencia en el centro a partir de la percepción que tienen los estudiantes de Enseñanza Secundaria obligatoria (ESO) y proponer la mediación escolar entre iguales como instrumento proactivo de gestión de conflictos.

Y como objetivo específico:

Comprobar en mayor o menor medida la presencia de los conflictos esbozados: Violencia Física Indirecta (VFI), Disrupción en el Aula (DA), Violencia Verbal de Alumnado-Alumnado (VVAA), Violencia Verbal AlumnadoProfesorado (VVAP), Violencia Física Directa entre estudiantes (VFDE), Violencia Física Indirecta (VFIA), Exclusión Social (ES) y Violencia TIC (VTIC). Realizando para ello un análisis de la frecuencia con la que el alumnado de 4응 de ESO, percibe la aparición de las diferentes conductas indeseadas, protagonizada por los estudiantes o el profesorado de su clase, en un centro educativo concertado de Málaga.

\section{Metodología}

Se ha llevado a cabo una metodología de encuestas, basada en la administración del CUVE3-ESO. Tal como dicen sus autores, este instrumento es un cuestionario diseñado para analizar la percepción de los estudiantes sobre la frecuencia de aparición de diferentes tipos de violencia escolar, protagonizada por el alumnado o el profesorado (Dobarro et al., 2016). El CUVE3-ESO adopta la forma de una escala tipo Likert compuesta por 44 ítems con 5 opciones de respuesta (desde 1 "Nunca" hasta 5 "Siempre).

Los análisis factoriales reportados por los autores, sobre una muestra de 2597 estudiantes de ESO, pertenecientes a 18 centros educativos de Asturias, mostraron que la estructura factorial que mejor ajusta a los datos es la compuesta por ocho factores: (1) violencia verbal de alumnado hacia alumnado; (2) violencia verbal de alumnado hacia profesorado; (3) violencia física directa y amenazas entre estudiantes; (4) violencia física indirecta por parte del alumnado; (5) exclusión social; (6) violencia a través de las tecnologías de la información y de la comunicación; (7) disrupción en el aula; y (8) violencia de profesorado hacia alumnado. (ver anexo, tabla 6). La fiabilidad de la escala en su conjunto fue de 0.939. Las escalas presentaban una fiabilidad alpha de Cronbach de entre 0.714 y 0.872 (Álvarez-García et al., 2013).

El cuestionario se responde en unos 15 a 20 minutos. Para el alumnado con alguna dificultad de comprensión lectora, se recurre a la ayuda del docente.

Para esta investigación se contó con la participación de una muestra incidental de 220 estudiantes de ESO de un centro ubicado en Málaga (España) en un contexto sociocultural medio-bajo. En la tabla 1 del anexo se proporcionan la frecuencia de participación con relación a la población de interés.

Tabla 1

Frecuencias de participación

\begin{tabular}{ccccc}
\hline Cursos & Frecuencia & Porcentaje & \% Válido & \% Acumulado \\
\hline 1ㅇ ESO & 54 & 24,50 & 24,50 & 24,50 \\
2o ESO & 56 & 25,50 & 25,50 & 50 \\
3 ESO & 57 & 25,90 & 25,90 & 75,90 \\
4을ㅇ & 53 & 24,10 & 24,10 & 100 \\
Total & 220 & 100 & 100 & \\
\hline
\end{tabular}

Fuente: propia datos obtenidos del centro curso 2017-2018 
La contestación al cuestionario ha sido siempre anónima y voluntaria. Se obtuvo el consentimiento informado de los participantes antes de iniciar la recogida de los datos y tras explicar el objetivo del estudio y el tratamiento que se iba a dar a la información.

Para realizar nuestro trabajo los cuestionarios fueron aplicados en enero, periodo del curso en donde hay ya ciertas relaciones establecidas en los alumnos de ESO.

1) Selección de la muestra

2) Recogida de datos

3) Análisis datos

Con objeto de evitar la deseabilidad social, se optó por que el encargado de supervisión de la prueba fuese alguien que no era docente del grupo y la contestación al cuestionario ha sido siempre anónima y voluntaria. Por ello, previamente se motivó al alumnado a participar en la evaluación, explicando brevemente en qué consistía y cuál era su objetivo último de la prueba -mejorar la convivencia en el centro-, con el fin de obtener el mayor número de respuestas sinceras. En el momento en el que los estudiantes entregaron el cuestionario cumplimentado, el evaluador comprobó que todos ellos habían respondido a todos las cuestiones planteadas.

Las variables para analizar tanto la percepción de las relaciones entre del alumnado como con el profesorado han sido: Disrupción (D), Violencia Verbal Alumnado-Alumnado (VVAA), Violencia Verbal Alumnado-Profesorado (VVAP), Exclusión Social (ES), Violencia Física Directa entre Estudiantes (VFDAE), Violencia Física Indirecta entre Estudiantes (VFIA), Violencia TIC (VTIC) y Violencia Profesor-Alumnado (VPA). Todas estas variables con sus correspondientes items aparecen descritas en la tabla 6 del Anexo.

Para iniciar el proceso de tratamiento de los datos, se procedió a un análisis de las propiedades psicométricas del instrumento con los datos de la presente muestra. Se calculó el coeficiente alpha de Cronbach de consistencia interna para cada escala del CUVE3-ESO junto con el coeficiente Omega de McDonald. Igualmente, se confirmó la estructura latente de la medición aplicando un análisis factorial confirmatorio según la estructura de ocho factores que indican (Dobarro et al., 2016) para valorar el ajuste del modelo se utilizaron los índices Chicuadrado con relación a sus grados de libertad, RMSEA, SRMR, TLI y CFI (Kaplan, 2009).

Posteriormente, se calcularon las puntuaciones de cada participante para cada uno de los factores. Para ello promediando sus puntuaciones en los ítems correspondientes a dichos factores. A partir de estos resultados se analizó el perfil de respuesta de los participantes de forma descriptiva para analizar la percepción de la frecuencia de conductas de violencia escolar, siguiendo el objetivo de estudio. Por último, se exploró la posible existencia de diferencias en la apreciación de aparición de conductas violentas, en función del género y del curso de los participantes. Para todo el proceso de análisis se utilizó la aplicación JASP (2018).

\section{Resultados}

Los coeficientes de consistencia interna para la escala global fueron de 0.921 para el alpha de Cronbach y de 0.923 para el Omega de McDonald. Los valores para las ocho subescalas se muestran en la tabla 2. Se puede observar que los valores son aceptables, aunque la escala VFIA obtiene un valor de consistencia interna alpha, bastante mediocre. Por tal motivo, la medida sobre dicha escala deberá interpretarse con cierta precaución a la hora de generalizar las conclusiones. 
Tabla 2

Coeficientes alfa y omega de las subescalas

\begin{tabular}{ccc}
\hline Escala & alfa & omega \\
\hline DA & 0.803 & 0.808 \\
VVAA & 0.778 & 0.786 \\
VVAP & 0.782 & 0.790 \\
ES & 0.680 & 0.696 \\
VFDAE & 0.720 & 0.732 \\
VFIA & 0.596 & 0.630 \\
VTIC & 0.828 & 0.830 \\
VPA & 0.853 & 0.857 \\
\hline
\end{tabular}

El análisis factorial confirmatorio sobre una estructura de ocho factores obtuvo un valor de Chi-cuadrado de 1832 con 874 grados de libertad, lo que supone una razón Chi-cuadrado/grados de libertad de 2.09, lo que puede considerarse como un ajuste aceptable. Lo mismo ocurre con los índices de ajuste RMSEA=0.07 y SRMR=0.078, que indican un ajuste aceptable (Shi et al., 2018). Por el contrario, los índices sugieren un ajuste bajo. Tomando en consideración todos los índices, se asume un ajuste moderado, aunque aceptable (Hormigo, 2014).

A partir de esta estructura se calcularon las puntuaciones ponderadas para cada participante, procediendo al análisis descriptivo de la muestra en las ocho dimensiones.

Los estadísticos descriptivos para las ocho dimensiones o factores se presentan en la tabla 3. En la misma, se puede observar que la frecuencia de disrupciones en el aula (factor DA) es intermedia (promedio de 3.52, error típico de la media de 0.064). Destaca que la puntuación mínima es de 1.33 y la máxima de 5 puntos, presentando un ligero sesgo negativo (-0.109).

Con relación a la violencia verbal del alumnado, el promedio de la frecuencia percibida también es intermedia (promedio $=3.03$; E.T. $=0.059$ ) presentando un sesgo positivo $(0.213)$ y una distribución bastante similar a la anterior dimensión.

Respecto a la violencia verbal del docente (factor VVAP) la puntuación promedio es algo más baja (2.63; E.T.=0.061) con una desviación típica de 0.917 lo que indica un acuerdo entre los participantes con relación a este fenómeno, similar a las respuestas en los factores anteriores.

Otro de los aspectos medidos es la exclusión social (ES). En este caso, la distribución de respuestas presenta un perfil distinto a los anteriores. Los participantes presentan una percepción de su existencia de 1.73 puntos de promedio $(E . T .=0.046)$ con una mediana de 1.50 puntos, aunque con algo menos de acuerdo entre las respuestas de los participantes (D.T. $=0.695)$.

En otro orden de cosas, la violencia directa entre el alumnado (VFDAE) presenta un promedio de 1.93 (E.T.=0.043) con una mediana de 1.80 puntos, lo que indica sesgo positivo que llega 1.03 puntos. En este mismo sentido, la violencia indirecta (VFIA) también presenta un promedio relativamente bajo $(1.65, E . T .=0.037)$ con una mediana de 1.60 puntos y una deviación típica de 0.552 que indica una mayor homogeneidad en la opinión de los participantes.

El factor que analiza la violencia relacionada con las nuevas tecnologías (VITC) también presenta la media más baja de todas las dimensiones con un promedio de 1.58 (E.T.=0.035) con una mediana de 1.45 y una deviación típica también la más baja (D.T.=0.527). 
Por último, la violencia de docentes hacia el alumnado se aprecia con un promedio de 2.11 puntos (E.T.=0.045) con una mediana de 2.05 , con un grado de homogeneidad similar a las otras dimensiones (D.T. $=0.681$ ) y un ligero sesgo positivo (sesgo=0.706).

En una siguiente fase, se exploraron posibles diferencias entre género. Puesto que en la distribución de las dimensiones no pueden considerarse normales (ver prueba Shapiro-Wilk en tabla 3) se aplicó la prueba KruskallWallis. Los resultados no mostraron diferencias significativas en función del género de los participantes a un nivel de confianza del $99 \%$. Solamente se obtuvo una diferencia significativa a un nivel de significación de 0.05 en la dimensión de violencia verbal entre el alumnado (VVAA). En concreto el valor K-W fue de 4.042 puntos con una probabilidad asociada de 0.044 y sólo un efecto de tamaño de 0.018 , por lo que este resultado los autores de este estudio consideran que no existe evidencia suficiente como para considerar que existan diferencias entre hombres y mujeres.

Tabla 3

Estadísticos descriptivos de los ocho factores estructurales

\begin{tabular}{ccccccccc}
\hline & DA & VVAA & VVAP & ES & VFDAE & VFIA & VTIC & VPA \\
\hline $\mathrm{N}$ & 220 & 220 & 220 & 220 & 220 & 220 & 220 & 220 \\
Missing & 0 & 0 & 0 & 0 & 0 & 0 & 0 & 0 \\
Mean & 3.52 & 3.03 & 2.63 & 1.73 & 1.93 & 1.65 & 1.58 & 2.11 \\
Std. error mean & 0.0642 & 0.0595 & 0.0618 & 0.0468 & 0.0434 & 0.0372 & 0.0355 & 0.0459 \\
Median & 3.67 & 3.00 & 2.67 & 1.50 & 1.80 & 1.60 & 1.45 & 2.05 \\
Standard deviation & 0.953 & 0.882 & 0.917 & 0.695 & 0.643 & 0.552 & 0.527 & 0.681 \\
Minimum & 1.33 & 1.25 & 1.00 & 1.00 & 1.00 & 1.00 & 1.00 & 1.00 \\
Maximum & 5.00 & 5.00 & 5.00 & 4.25 & 4.40 & 3.60 & 3.20 & 5.00 \\
Skewness & -0.109 & 0.213 & 0.343 & 1.25 & 1.03 & 0.868 & 0.885 & 0.706 \\
Std. error skewness & 0.164 & 0.164 & 0.164 & 0.164 & 0.164 & 0.164 & 0.164 & 0.164 \\
Kurtosis & -0.990 & -0.848 & -0.294 & 1.56 & 1.35 & 0.201 & 0.107 & 0.958 \\
Std. error kurtosis & 0.327 & 0.327 & 0.327 & 0.327 & 0.327 & 0.327 & 0.327 & 0.327 \\
Shapiro-Wilk $\mathrm{p}$ & $<.001$ & $<.001$ & $<.001$ & $<.001$ & $<.001$ & $<.001$ & $<.001$ & $<.001$ \\
\hline
\end{tabular}

En la siguiente fase del proceso de análisis se indagó en las posibles diferencias entre cursos, lo supone analizar posibles diferencias en función de la edad. En este caso, se registran diferencias significativas en todas las dimensiones excepto en exclusión social (ES) tal como puede comprobarse en la prueba de Kruskal-Wallis de la tabla 4.

Tabla 4

Prueba Kruskal-Wallis de diferencia entre cursos

\begin{tabular}{lcccc}
\hline & $\chi^{2}$ & $\mathrm{df}$ & $\mathrm{p}$ & $\varepsilon^{2}$ \\
\hline DA & 62.58 & 3 & $<.001$ & 0.2858 \\
VVAA & 69.03 & 3 & $<.001$ & 0.3152 \\
VVAP & 100.51 & 3 & $<.001$ & 0.4589 \\
ES & 2.53 & 3 & 0.471 & 0.0115 \\
VFDAE & 19.99 & 3 & $<.001$ & 0.0913 \\
VFIA & 15.04 & 3 & 0.002 & 0.0687 \\
VTIC & 37.13 & 3 & $<.001$ & 0.1696 \\
VPA & 58.74 & 3 & $<.001$ & 0.2682 \\
\hline
\end{tabular}


Para indagar las diferencias en concreto, se calcularon los estadísticos descriptivos del promedio, desviación típica y error típico por curso y dimensión (tabla5).

Estos resultados presentan un patrón común en forma de arco invertido en todas las dimensiones que consiste en un valor medio más bajo en primer curso, para experimentar un incremento en segundo y tercero y volver a bajar en cuarto curso (excepto en violencia física indirecta VFIA que se mantiene en el mismo nivel que el tercero). A este patrón, se escapa la exclusión social (ES) que no tiene diferencias significas, presentando una tendencia en U. En cualquier caso, las diferencias son estadísticamente significativas, aunque los tamaños de efecto son relativamente bajos.

Tabla 5

Estadísticos descriptivos de los ocho factores estructurales

\begin{tabular}{|c|c|c|c|c|c|}
\hline & IdCurso & $\mathrm{N}$ & Media & D.T. & E.T. \\
\hline \multirow[t]{4}{*}{ DA } & $10 \mathrm{ESO}$ & 54 & 3.06 & 0.866 & 0.1178 \\
\hline & $2 \circ \mathrm{ESO}$ & 56 & 3.65 & 0.778 & 0.1040 \\
\hline & 3 ESO & 57 & 4.26 & 0.751 & 0.0994 \\
\hline & 40 ESO & 53 & 3.04 & 0.860 & 0.1181 \\
\hline \multirow[t]{4}{*}{ VVAA } & 1으 ESO & 54 & 2.51 & 0.681 & 0.0926 \\
\hline & $20 \mathrm{ESO}$ & 56 & 2.84 & 0.696 & 0.0931 \\
\hline & 3으 ESO & 57 & 3.84 & 0.645 & 0.0855 \\
\hline & $40 \mathrm{ESO}$ & 53 & 2.88 & 0.889 & 0.1221 \\
\hline \multirow[t]{4}{*}{ VVAP } & 1을 & 54 & 1.75 & 0.501 & 0.0682 \\
\hline & $20 \mathrm{ESO}$ & 56 & 2.98 & 0.773 & 0.1033 \\
\hline & 3으 ESO & 57 & 3.32 & 0.770 & 0.1020 \\
\hline & 4으 ESO & 53 & 2.41 & 0.712 & 0.0978 \\
\hline \multirow[t]{4}{*}{ ES } & 1으 ESO & 54 & 1.81 & 0.808 & 0.1100 \\
\hline & 20 ESO & 56 & 1.73 & 0.562 & 0.0751 \\
\hline & 3으 ESO & 57 & 1.64 & 0.696 & 0.0922 \\
\hline & 40 ESO & 53 & 1.76 & 0.704 & 0.0967 \\
\hline \multirow[t]{4}{*}{ VFDAE } & 1을 & 54 & 1.84 & 0.594 & 0.0808 \\
\hline & $20 \mathrm{ESO}$ & 56 & 2.02 & 0.634 & 0.0848 \\
\hline & 3으 ESO & 57 & 2.20 & 0.753 & 0.0997 \\
\hline & 40 ESO & 53 & 1.65 & 0.413 & 0.0567 \\
\hline \multirow[t]{4}{*}{ VFIA } & 1으 ESO & 54 & 1.44 & 0.416 & 0.0566 \\
\hline & 2ㅇ ESO & 56 & 1.61 & 0.604 & 0.0806 \\
\hline & 3으 ESO & 57 & 1.76 & 0.573 & 0.0759 \\
\hline & 40 ESO & 53 & 1.78 & 0.540 & 0.0741 \\
\hline \multirow[t]{4}{*}{ VTIC } & 1으 ESO & 54 & 1.34 & 0.402 & 0.0547 \\
\hline & 20 ESO & 56 & 1.50 & 0.492 & 0.0657 \\
\hline & 3으 ESO & 57 & 1.92 & 0.531 & 0.0703 \\
\hline & 40 ESO & 53 & 1.54 & 0.501 & 0.0688 \\
\hline \multirow[t]{4}{*}{ VPA } & 1 으응 & 54 & 1.70 & 0.558 & 0.0759 \\
\hline & 20 ESO & 56 & 1.88 & 0.473 & 0.0632 \\
\hline & 3으 ESO & 57 & 2.61 & 0.656 & 0.0869 \\
\hline & $40 \mathrm{ESO}$ & 53 & 2.24 & 0.646 & 0.0888 \\
\hline
\end{tabular}


Hemos observado con relación a la violencia verbal del alumnado, que el promedio de la frecuencia percibida es intermedia (promedio= 3.03; E.T.=0.059) presentando un sesgo positivo (0.213). La violencia verbal entre el alumnado (factor VVAA) que, aunque la frecuencia no es muy alta, este tipo de violencia es una realidad en el aula y que la diferencia con la violencia verbal hacia docente (factor VVAP) es menor que a la del alumnado. La violencia verbal del docente (factor VVAP) cuya puntuación promedio es algo más baja $(2.63 ; \mathrm{E} . \mathrm{T} .=0.061$ ) con una desviación típica de 0.917, nos indica un acuerdo entre los participantes con relación a este fenómeno, similar a las respuestas en otros factores. Lo que se concluye que la mayoría de los estudiantes tienen un nivel de percepción bajo de la violencia verbal tanto entre estudiantes como de estudiantes hacia el profesorado. En la mayoría de los casos estas conductas están completamente normalizadas, suelen ser habituales y cotidianas. Los resultados son similares a los obtenidos en otros estudios realizados (Nieto et al., 2018 \& Zúñiga et al., 2019) y hacen reflexionar de que no se toma conciencia de que estas conductas son actos violentos hacia los compañeros y compañeras.

Otro de los aspectos evaluado es la exclusión social en las aulas y centro escolar (Factor ES). En este caso, la distribución de respuestas presenta un perfil distinto a los anteriores. Los participantes presentan una percepción de su existencia de 1.73 puntos de promedio (E.T.=0.046) con una mediana de 1.50 puntos, aunque con algo menos de acuerdo entre las respuestas de los participantes (D.T.=0.695). En nuestro estudio señalamos que los estudiantes no perciben este conflicto en las aulas ni en el entorno y está menos presente que otros factores. Esto no sucede en otros estudios realizados (Sandoval et al., 2017). La causa de estas diferencias de percepción puede estar en distintas cuestiones, el número de alumnos, la ubicación del centro, la diferencia de ideario de centro y en el tipo de alumnado que asiste al mismo.

En otro orden de cosas, la violencia directa entre el alumnado (VFDAE) presenta un promedio de 1.93 (E.T.=0.043) con una mediana de 1.80 puntos, lo que indica sesgo positivo que llega 1.03 puntos. En este mismo sentido, la violencia indirecta (VFIA) también presenta un promedio relativamente bajo $(1.65$, E.T. $=0.037)$ con una mediana de 1.60 puntos y una deviación típica de 0.552 que indica una mayor homogeneidad en la opinión de los participantes. Si comparamos la violencia física directa con la violencia verbal observamos que esta violencia verbal es relativamente mayor que la física, incluso que la disrupción en el aula. Hemos verificado que tanto la violencia verbal como la disrupción en el aula son los conflictos más cotidianos. Otros estudios manifiestan esta realidad en las aulas (Gutiérrez-Nieves, 2019).

El impacto que tienen las tecnologías de la información y la comunicación (TIC) es una frecuente preocupación en el marco escolar (Dominguez-Alonso \& Portela, 2020). El factor que analiza la violencia relacionada con las nuevas tecnologías (factor VITC) también presenta la media más baja de todas las dimensiones con un promedio de 1.58 (E.T.=0.035) con una mediana de 1.45 y una deviación típica también la más baja (D.T.=0.527). Curiosamente, hemos observado que este factor VTIC a través de telefonía móvil u otros aparatos electrónicos usados para redes sociales o mensajería instantánea es el menor de todos. Actualmente, con la avalancha de casos que se dan con este tipo de violencia llegando en ocasiones al ciberacoso. La violencia TIC se está generalizando en la población juvenil según los últimos estudios realizado por Domínguez-Alonso \& Portela, (2020). Lo que nos ha llamado la atención es que en nuestra investigación el resultado obtenido es el menor de entre todos los factores analizados. Bien es cierto, que en los últimos años se está haciendo hincapié en la prohibición de usos de móviles en el centro y el alumnado toma conciencia del tipo de daño que es capaz de causar con las TIC.

\section{Conclusiones}

Generalmente la violencia familiar de reproduce en la escuela (Pazo, 2020) y en ambos casos esta violencia afecta negativamente al rendimiento académico y al desarrollo psicosocial del alumnado, siendo un factor de riesgo en 
el rendimiento académico y en la deserción escolar (Álvarez-Gómez, 2019; Zepeda, 2020 ). Respecto al rendimiento académico, se ha observado que participar como agresor en situaciones de maltrato entre estudiantes, permite predecir una baja percepción de competencia académica por parte del propio alumno y la obtención de bajas calificaciones (Ma, 2008), así como abandono escolar (Sancho et al., 2014). En este mismo sentido, se ha apreciado que el comportamiento disruptivo en el aula por parte del alumnado muestra una correlación inversa con el apoyo por parte del profesorado y con el logro académico. Ser rechazado y víctima de agresiones genera ansiedad y también afecta al logro académico y al riesgo de fracaso escolar y, en muchos casos, evitación escolar, absentismo, pérdida del compromiso con lo académico y muy baja participación, frecuentemente para evitar el maltrato (Juvonen et al., 2000; Bush et al., 2006; Totura et al., 2009 ).

La relación con el profesorado también incide en el rendimiento académico. El fomento de relaciones cercanas y de calidad entre estudiantes y formadores está asociado a un mayor compromiso en el centro educativo por parte de ambos (Anderson et al., 2004), lo cual se traduce en una mayor motivación, rendimiento, sentimientos de pertenecía y afecto en la escuela.

Se puede considerar que la violencia escolar también puede tener un impacto negativo entre los implicados sobre ciertos aspectos de carácter psicosocial, Así, los problemas de comportamiento por parte del alumnado constituyen una de las principales fuentes de estrés y burnout en el profesorado, lo que puede derivar no sólo en insatisfacción y desmotivación laboral, sino también, en síntomas de tipo somático, depresivo, de ansiedad, insomnio o irritabilidad, actitudes negativas hacia uno mismo o hacia los demás -que pueden afectar también a su vida familiar-, e incluso en casos más extremos fobia social, depresiones graves o intentos de suicidio (Moriana \& Herruzo, 2004).

Los estudiantes que han sido víctimas de un maltrato continuado por parte de sus compañeros pueden mostrar trastornos del sueño, pérdida de apetito y ansiedad, desconfianza ante los demás, ansiedad social, tendencia al aislamiento, una autoestima baja, autoconcepto negativo, una mayor vulnerabilidad a la depresión de lo habitual y, en los casos más graves, llegar a presentar ideas de suicidio (Romualdo et al., 2019). Por último, el alumnado que presenta problemas de comportamiento en el centro educativo puede extender su conducta a otros ámbitos, como el ámbito laboral, la pareja o el barrio.

A la luz de los resultados, la primera evidencia que surge de este trabajo de investigación es que el fenómeno de la violencia escolar es una realidad patente y relevante en el centro educativo concertado donde se ha realizado el estudio. Se corrobora la presencia de DA, VVAA, VVAP, ES, VFDAE, VFIA, VTIC, VPA, algunos como hemos visto en los resultados en mayor proporción y significatividad, lo que permite corroborar el objetivo especifico planteado en el estudio.

Del estudio se desprende un elemento francamente relevante, y es que al contrario de lo que pudiera parecer y así se halla en otros estudios la calidad de la convivencia se merma en los últimos cursos de la Educación Secundaria, esto es, se incrementa la conflictividad en los últimos años de la ESO (Domínguez-Alonso et al., 2019; Medina \& Reverte, 2019).

Nuestra propuesta para disminuir los niveles de violencia escolar en estos cursos de ESO es la implementación de la mediación entre iguales. Es un proceso formal, libre, confidencial y voluntario que busca la gestión pacífica de los conflictos y que promueve que las partes en conflicto negocien un acuerdo y reconstruyan sus relaciones deterioradas (Boqué, 2018). La mediación supone una acción preventiva de participación, inclusión y corresponsabilidad e incluso reconocimiento de la autoridad del profesorado cuando estos participan en su promoción (Jares, 2006, Rotila, 2019) y repercute con el tiempo en la mejora de la convivencia escolar ya que la mediación escolar incentiva valores como la cooperación, comunicación, tolerancia y autocontrol (Pérez-Serrano \& Pérez-de-Guzmán, 2011), fomentando el respeto, diálogo, la empatía, el compromiso y la responsabilidad 
necesarias para la mejora del ambiente educativo. En síntesis, se trata de articular activamente una cultura de la mediación, entendiéndola como una oportunidad para la generación de puentes prácticos, innovadores y para la inclusión educativa.

\section{Referencias bibliográficas}

Álvarez-García, D., Álvarez, L., Nuñez, J. C., González-Pienda, J. A., González-Castro, P., \& Rodríguez, C. (2008). Estudio del nivel de violencia escolar en siete centros asturianos de Educación Secundaria. Aula Abierta, $36,89-96$.

Álvarez-García, D., Nuñez, J. C., \& Dobarro, A. (2013). Cuestionarios para evaluar la violencia escolar en Educación Primaria y en Educación Secundaria: CUVE3-EP y CUVE3-ESO. Apuntes de Psicología, 31-2, 191202. https://doi.org/https://doi.org/10.17060/ijodaep.2014.n1.v5.710

Álvarez-Gómez, B. P. (2019). Violencia escolar y el desarrollo socio afectivo de los niños y niñas de la unidad educativa unidad popular periodo 2019 [BABAHOYO: UTB, 2019]. http://dspace.utb.edu.ec/handle/49000/7146

Anderson, A. R., Christenson, S. L., Sinclair, M. F., \& Lehr, C. A. (2004). The importance of relationships for promoting engagement with school. Journal of School Psychology, 42, 95-113. https://doi.org/https://doi.org/10.1016/j.jsp.2004.01.002

Andrades-Moya, J. (2020). Convivencia escolar en Latinoamérica: Una revisión bibliográfica. Revista Electrónica Educare, 24 (2)(2), 19-32. https://doi.org/10.15359/ree.24-2.17

Arón, A. M. ., Milicic, N., Sánchez, M., \& Subercaseux, J. (2017). Construyendo juntos: claves para la convivencia escolar. https://educrea.cl/wp-content/uploads/2018/11/DOC2-convivencia_escolar.pdf

Ascorra, P., López, V., Núñez, C. G., Bilbao, M. Á., Gómez, G., \& Morales, M. (2016). Relación entre segregación y convivencia escolar en escuela públicas chilenas. Universitas Psychologica, 15(1). https://doi.org/10.11144/Javeriana.upsy15-1.rsce

Boqué, M. C. (2018). La mediación va a la escuela. Hacia un buen plan de convivencia en el centro. Narcea.

Brandoni, F. (2017). Conflictos en la escuela. EDUNTREF. http://eduntref.com.ar/magento/pdf/conflictos-en-laescuela-digital.pdf

Bush, E. S., Ladd, G. W., \& Herald, S. L. (2006). Peer exclusion and victimization: processes that mediate the relation between perr group rejection and children's clasroom engagement and achievement? Journal of Educational Psychology, 98(1), 1-13. https://doi.org/https://doi.org/10.1037/0022-0663.98.1.1

Campos, J. A., Tass, K. O. M. El, \& Cruz, G. de C. (2015). Violência escolar: relações entre bullying e a educação física. Revista Espacios, 36(11), E-1. https://www.revistaespacios.com/a15v36n11/153611E1.html

Costa, V., Teyes, R., \& Zamora., M. (2020). Haciendo ciencia construimos futuro. In Haciendo ciencia, construimos futuro (pp. 580-592). Universidad de Zulia.

https://scholar.google.es/scholar?hl=es\&as_sdt=0\%2C5\&q=Haciendo+ciencia+construimos+futuro+Teyes \&btnG $=$

Delors, J. (1996). Los cuatro pilares de la educación. Informe a la UNESCO de la Comisión internacional sobre la educación para el siglo XXI. (S. UNESCO (ed.)). http://192.188.48.56/ojs/index.php/galileo/articl

Dobarro, A., Álvarez García, D., \& Núñez, J. C. (2016). CUVE3: Instrumentos para evaluar la violencia escolar. International Journal of Developmental and Educational Psychology. Revista INFAD de Psicología., 5(1), 487-492. https://doi.org/10.17060/ijodaep.2014.n1.v5.710 
Domínguez-Alonso, J., López-Castedo, A., \& Nieto-Campos, B. (2019). Violencia escolar: diferencias de género en estudiantes de secundaria. Revista Complutense de Educación, 30(4), 1031-1044.

https://doi.org/https://doi.org/10.5209/rced.59997

Dominguez-Alonso, J., \& Portela Pino, I. (2020). Violencia a través de las TIC: comportamientos diferenciados por género. RIED. Revista Iberoamericana de Educación a Distancia, 23(2), 273. https://doi.org/10.5944/ried.23.2.25916

Estrada, E. G., \& Mamani, H. J. (2020). Violencia escolar y niveles de logro de aprendizaje en una institución educativa pública de Puerto Maldonado. PURIQ, 2(3), 246-260. https://doi.org/10.37073/puriq.2.3.86

Fierro-Evans, C., \& Carbajal-Padilla, P. (2019). Convivencia Escolar: Una revisión del concepto. Psicoperspectivas, 18(1), 9-27. https://doi.org/10.5027/psicoperspectivas-Vol18-Issue1-fulltext-1486

Garaigordobil, M. y Oñederra, J. A. (2010). (2010). La violencia entre iguales. Revisión teórica y estrategias de intervención. Piramide.

Garaigordobil, M. (2019). Prevention of cyberbullying: personal and family predictive variables of cyberaggression Prevención del cyberbullying: variables personales y familiares predictoras de ciberagresión. Revista de Psicología Clínica Con Niños y Adolescentes, 6(3), 2019-2028. https://doi.org/10.21134/rpcna.2019.06.2.1

Gutiérrez-Nieves, A. (2019). Tipos de violencia escolar percibidos por futuros educadores y la relación de las dimensiones de la Inteligencia Emocional. Interacciones: Revista de Avances En Psicología, 5 (2)(2), e150. https://doi.org/https://doi.org/10.24016/2019.v5n2.150

Hormigo, A. L. (2014). Introducción a las ecuaciones estructurales en AMOS y R. https://masteres.ugr.es/moea/pages/curso201314/tfm1314/tfmseptiembre1314/memoriamasterantonio_lara_hormigo/!

Hulac D.H. y Benson, N. (2010). The use of group contingencies for preventing and managing disruptive behaviors. Intervention in School \& Clinic, 45(4), 257-262.

https://doi.org/https://doi.org/10.1177/1053451209353442

Jares, X. R. (2006). Pedagogía de la convivencia. Graó.

JASP. (2018). A New Manual for JASP - JASP - Free and User-Friendly Statistical Software. https://jaspstats.org/2018/09/13/a-new-manual-for-jasp/

Jurado de los Santos, P., Lafuente Carrasco, Á., \& Justiniano Domínguez, M. D. (2020). Conductas disruptivas en Educación Secundaria Obligatoria: análisis de factores intervinientes. Contextos Educativos. Revista de Educación, 25(25), 219-236. https://doi.org/10.18172/con.3827

Jurado, P., \& Justiniano, M. D. (2016). Propuestas de intervención ante las conductas disruptivas en Educación Secundaria Obligatoria. Revista Española de Orientación y Psicopedagogía (REOP), 27(3), 8-25. https://www.redalyc.org/pdf/3382/338250662002.pdf

Juvonen, J., Nishina, A., \& Graham, S. (2000). Perr haressment, psychological adjustent and school functioning in early adolescence. Jounal of Educational Psychology, 92(2), 349-359.

https://doi.org/https://doi.org/10.1037/0022-0663.92.2.349

Kaplan, D. (2009). Structural equation modeling: foundations and extensions. Segunda edición. Thousand Oaks, CA: Sage. https://doi.org/doi:10.4135/9781452226576.

Ma, L. (2008). The development of academic competence amon adolescente who bully an who ara bullied. Disertation Abstract International: Section B: The Sciences and Engineering, 68(7-B), 4866. 
Medina, J. A., \& Reverte, M. J. (2019). Violencia escolar, rasgos de prevalencia en la victimización individual y grupal en la Educación Obligatoria en España. Revista de Estudios y Experiencias En Educación, 18(37), 97110. https://doi.org/10.21703/rexe.20191837medina9

Medina, M. A. G., \& Villarreal, D. C. T. (2019). Violencia escolar en bachillerato: algunas estrategias para su prevención desde diferentes perspectiva Educación. Teoría de La Educación. Revista Interuniversitaria USAL., 123-147. https://doi.org/DOI: http://dx.doi.org/10.14201/teri.19616

Moriana, J.A., y Herruzo, J. (2004). Estrés y burnout en profesores . International Journal of Clinical and Health Psychology, 4(3), 597-621. https://www.redalyc.org/pdf/337/33740309.pdf

Nieto, B., Portela, I., López, E., \& Domínguez, V. (2018). Violencia verbal en el alumnado de Educación Secundaria Obligatoria. European Journal of Investigation in Health, Psychology and Education, 8(1), 5. https://doi.org/10.30552/ejihpe.v8i1.221

Novoa, M., Reina, R. Á., \& Yerovi, E. J. (2020). Cultura de Paz Como Mecanismo para la Convivencia Humana, Intercultural y de otras Nacionalidades, desde la Educación. Revista de Investigación, Formación y Desarrollo: Generando Productividad Institucional, 8(1), 7. https://doi.org/10.34070/rif.v8i1.186

Ormart, E. B. (2019). Violencia escolar y planificación educativa - Dialnet. Nodos y Nudos: Revista de La Red de Calificación de Educadores, Vol. 6,(46). https://dialnet.unirioja.es/servlet/articulo?codigo=7143984

Pachter, L. M., Bernstein, B. A., Szalacha, L. A., \& Coll, C. G. (2010). Perceived racism and discrimination in children and youths: An exploratory study. Health \& Social Work, 35(1), 61-70. https://doi.org/https://doi.org/10.1093/hsw/35.1.61

Pazo, J. (2020). Entre la violencia familiar y la violencia escolar. Investigaciones Sociales, 22(42), 19-36. https://doi.org/10.15381/is.v22i42.17477

Pérez-Serrano, G., \& Pérez-de-Guzmán, M. V. (2011). Aprender a convivir: El conflicto como oportunidad de crecimiento. Narcea.

Rizo-Arias, L. J. (2019). Índices de exclusión educativa en el etapa de la ESO en la provincia de Salamanca. Papeles Salmantinos de Educación, 23, 9-29.

https://revistas.upsa.es/index.php/papeleseducacion/article/view/21

Romualdo, C., Abadio de Oliveira, W., da Silva, J. L., Cuadros Jiménez, O. E., \& lossi Silva, M. A. (2019). Papeles, características y consecuencias del acoso escolar entre estudiantes observadores: una revisión integradora de la literatura. Salud \& Sociedad, 10(1), 66-78. https://doi.org/10.22199/s07187475.2019.0001.00005

Rotila, A.-N. (2019). Objetivos de la Educación para la paz. In Estudios interdiciplinarios: Paz y comunicación. (pp. 378-398). https://n9.cl/87h4

Sánchez, A., Alexander, R., López, A., \& Patricia, A. (2019). La educación en mediación escolar como escenario de formación ciudadana Education in school mediation as a scenario for citizen education Contenido: Vol. 40 (21). https://www.revistaespacios.com/a19v40n21/a19v40n21p01.pdf

Sancho, J. M., Correa Gorospe, J. M., Giró Gràcia, X., \& Fraga, L. (2014). Aprender a ser docente en un mundo en cambio. Simposio internacional. http://hdl.handle.net/2445/50680

Sandoval, J., Abril, A., \& Leal, H. (2017). Violencia en la escuela: creencias y percepciones de docentes y estudiantes. http://www.comie.org.mx/congreso/memoriaelectronica/v14/doc/1895.pdf

Shi, D., Maydeu-Olivares, A., \& DiStefano, C. (2018). The Relationship Between the Standardized Root Mean Square Residual and Model Misspecification in Factor Analysis Models. Multivariate Behavioral Research, 53(5), 676-694. https://doi.org/10.1080/00273171.2018.1476221 
Tokunaga, R. S. (2010). Following you home from school: A critical review and synthesis of research on cyberbullying victimization. Computers in Human Behavior, 26(3), 277-287.

https://doi.org/https://doi.org/10.1016/j.chb.2009.11.014

Torrego, J. C. (2006). Desde la mediación de conflictos en centros escolares hacia el modelo integrado de mejora de la convivencia. In En J. C. Torrego (Coord.), Modelo integrado de mejora de la convivencia. Estrategias de mediación y tratamiento de conflictos (pp. 11-26). Graó.

Totura, C. M., Green, A. E., Karver, M. S., \& Gesten, E. L. (2009). Multiple informants in the assessment of psychological, behavior, and academic correllates of bullying and victimization in middle school. Jornal of Adolescense, 32, 193-211. https://doi.org/https://doi.org/10.1016/j.adolescence.2008.04.005

Uruñuela, P. M. (2018). Trabajar la convivencia en los centros educativos. Una mirada al bosque de la convivencia. . Narcea.

Vidal, R. G., Raga, L. G., \& Martín, R. L. (2019). Percepciones sobre la escuela democrática en Argentina y España. Educ. Pesqui. https://doi.org/https://doi.org/10.1590/s1678-4634201945188681

Vizcarra, M. . T., Rekalde, I., \& Macazaga, A. M. . (2018). La percepción del conflicto escolar en tres comunidades de aprendizaje. Magis. Revista Internacional de Investigación En Educación, Vol. 10 Nú, Pág. 95-108. https://dialnet.unirioja.es/servlet/articulo?codigo=6516365

Zepeda, M. de los Á. C. (2020). Los conflictos escolares como factor de riesgo en el rendimiento académico y la deserción escolar. Revista RedCA, v. 3, n. 7, pág. 82-100.

https://revistaredca.uaemex.mx/article/view/14703

Zúñiga, L. F. S., Rivas, P. L., \& Trevizo, J. G. R. (2019). Percepción de la violencia escolar en el último ciclo de educación primaria. Revista Electrónica Cientifica de Investigación Educativa, Vol. 4 Núm, 1349-1350. https://www.rediech.org/ojs/2017/index.php/recie/article/view/369 


\section{Anexos}

Tabla 6

Descriptores de ítems de Conflictos

Conflicto: Disrupción en el aula

da1 El alumnado dificulta las explicaciones del profesor/a con su comportamiento en clase

da2: $\quad$ Hay alumnado que ni trabaja ni deja trabajar al resto

da3: $\quad$ El alumnado dificulta las explicaciones del profesorado hablando en clase.

Conflicto: Violencia verbal entre alumnos

vvaa1: $\quad$ El alumnado pone motes molestos a sus compañeros/as.

vvaa2 El alumnado pone motes a sus compañeros o compañeras.

vvaa3 Hay estudiantes que extienden rumores negativos acerca de compañeros

vvaa4 Los estudiantes hablan mal unos de otros.

Conflicto: Violencia verbal de alumnado hacia el profesorado

vvap1 El alumnado habla con malos modales al profesorado.

vvap2

vvap3

El alumnado falta el respeto a los profesores en el aula.

Los estudiantes insultan a profesores y profesoras.

Conflicto: Exclusión social

es1

es2

es3

es4

Algunos estudiantes son discriminados por sus compañeros/as por sus buenos resultados académicos Determinados estudiantes son discriminados por sus compañeros/as por sus bajas notas.

Hay estudiantes que son discriminados por sus compañeros/as por diferencias culturales, étnicas o religiosas

Algunos estudiantes son discriminados por sus compañeros por su nacionalidad.

Conflicto: Violencia física directa y amenazas entre estudiantes

vfdae1

Los estudiantes amenazan a otros de palabra para meterles miedo u obligarles a hacer cosas.

vfdae2 Determinados estudiantes pegan a sus compañeros/as dentro del recinto escolar

vfdae3

Algunos estudiantes amenazan a otros/as con navajas u otros objetos para meterles miedo $u$ obligarles a hacer cosas

vfdae4

vfdae5

Algunos alumnos/as protagonizan agresiones físicas en las cercanías del recinto escolar.

El alumnado protagoniza peleas dentro del recinto escolar

Conflicto: Violencia física indirecta

vfia1 Ciertos estudiantes roban objetos o dinero del centro educativo

vfiae2 Ciertos estudiantes roban objetos o dinero de otros compañeros

vfiae3

Algunos estudiantes esconden pertenencias o material del profesorado

vfiae4

Algunos estudiantes toban cosas del profesorado

vfiae5

Determinados estudiantes causan desperfectos intencionadamente en pertenencias del profesorado.

Conflicto: Violencia TIC

vtic1

Hay estudiantes que envían a sus compañeros a través del correo electrónico, de ofensas, insulto o

amenazas

vtic2 Algunos estudiantes graban o hacen fotos a compañeros.

vtic3

Hay estudiantes que publican comentarios en Tuenti, Facebook, Instagram, de ofensa, insulto o amenaza acerca de compañeros

vtic4 Los estudiantes publican en internet fotos o videos ofensivos de compañeros

vtic5

Hay estudiantes que graban o hacen fotos a compañeros/as con el móvil para amenazarlas o chantajearlas.

vtic6 Hay estudiantes que graban y hacen fotos a profesores/as con el móvil para burlarse.

Ciertos estudiantes publican comentarios en Tuenti, Facebook, Instagram de ofensa, insulto o amenaza a profesores.

vtic8 Algunos estudiantes envían mensajes en Tuenti, Facebook, Instagram. de ofensa, insulto o amenazas.

vtic9 Los estudiantes publican en internet fotos o videos ofensivos de compañeros 
vtic10 Los estudiantes publican en internet fotos o videos ofensivos de profesores/as.

Conflicto: Violencia del profesor hacia los estudiantes

vpa1 Algunos estudiantes son discriminados por sus compañeros/as por sus buenos resultados académicos.

vpa2 El profesorado tiene manía a algunos alumnos/as.

vpa3 El profesorado ridiculiza al alumnado.

vpa4 El profesorado ignora a cierto alumnado.

vpa5 El profesorado castiga injustamente.

vpa6 El profesorado baja la nota a algún alumno como castigo.

vpa7 Ciertos profesores intimidan o atemorizan a algún alumno/a,

vpa8 Hay profesores que insultan al alumnado.

vpa9 El profesorado amenaza algún alumno

vpa10 El profesorado no escucha a su alumnado

Esta obra está bajo una Licencia Creative Commons Attribución-NoCommercial 4.0 International

(cc) BY-NC 